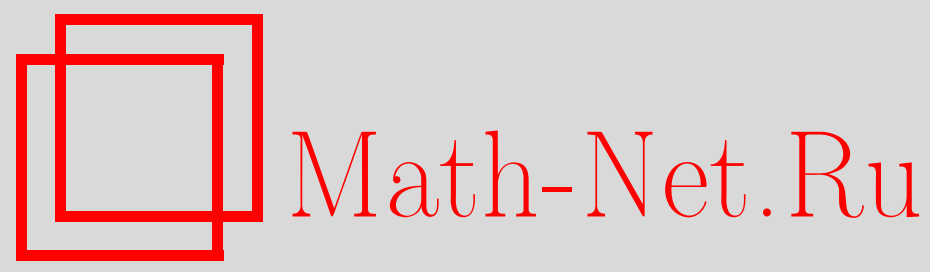

В. А. Емеличев, В. В. Коротков, Устойчивость векторной инвестиционной булевой задачи с критериями Вальда, Дискрет. матем., 2012, том 24, выпуск 3, 3-16

DOI: https://doi.org/10.4213/dm1192

Использование Общероссийского математического портала Math-Net.Ru подразумевает, что вы прочитали и согласны с пользовательским соглашением http://www.mathnet.ru/rus/agreement

Параметры загрузки:

IP : 52.205 .19 .152

26 апреля 2023 г., 15:31:08 
УДК 519.8

\title{
Устойчивость векторной инвестиционной булевой задачи с критериями Вальда
}

\author{
() 2012 г. В. А. Емеличев, В. В. Коротков
}

\begin{abstract}
Указаны нижняя и верхняя достижимые оценки радиуса устойчивости векторного булева варианта задачи портфельной оптимизации Марковица с максиминными критериями эффективности Вальда и паретовским принципом оптимальности.

Работа выполнена при поддержке Белорусского республиканского фонда фундаментальных исследований, проект Ф11К-095.
\end{abstract}

\section{1. Введение}

В [1], на основе портфельной теории Марковица, была сформулирована векторная инвестиционная булева модель с минимаксными критериями рисков Сэвиджа. Получены оценки радиуса устойчивости парето-оптимального портфеля. В настоящей статье в рамках модели Марковица рассматривается векторная задача формирования портфеля инвестора, максимизирующего различные виды эффективности портфеля в условиях нестабильности экономической ситуации и неопределенности исходных данных задачи. Исследуется тот тип устойчивости инвестиционной задачи к возмущениям ее параметров, который является дискретным аналогом свойства полунепрерывности сверху по Хаусдорфу многозначного отображения, переводящего любой набор параметров инвестиционной задачи в соответствующее множество Парето. При этом непредсказуемость состояния рынка учитывается путем использования максиминных критериев Вальда. В результате проведенного параметрического анализа найдены нижняя и верхняя достижимые (неулучшаемые) оценки радиуса устойчивости задачи для случая линейной нормы $l_{1}$ в пространстве портфелей и чебышевской нормы $l_{\infty}$ в критериальном пространстве и в пространстве состояний рынка. Отметим, что при таких же сочетаниях норм $l_{1}$ и $l_{\infty}$ в [2] анонсированы аналогичные оценки (сверху и снизу) радиуса устойчивости векторной инвестиционной булевой задачи с минимаксными критериями рисков Сэвиджа и паретовским принципом оптимальности.

\section{2. Постановка задачи}

Рассмотрим векторный дискретный вариант задачи управления инвестициями Марковица [3]. Для этого введем ряд обозначений и определений. Пусть $N_{n}=\{1,2, \ldots, n\}-$ альтернативные инвестиционные проекты (активы); $N_{m}$ - возможные состояния рынка 
(рыночные ситуации); вектор $x=\left(x_{1}, x_{2}, \ldots, x_{n}\right)^{T} \in X \subseteq \mathbf{E}^{n}-$ инвестиционный портфель с компонентами $x_{j}=1$, если инвестиционный проект $j \in N_{n}$ реализуется, и $x_{j}=0$ в противном случае. Здесь $\mathbf{E}=\{0,1\}$.

Существует несколько подходов к оценке экономической эффективности инвестиционных проектов (NPV, NFV, PI и др.), по разному учитывающих влияние факторов неопределенности и риска (см., например, $[4,5,6])$. Пусть $N_{s}$ - виды (показатели) эффективности проекта. Инвестиционный портфель $x$ будем оценивать величиной $\sum_{j \in N_{n}} e_{i j k} x_{j}$, где $e_{i j k}$ - ожидаемая оценка эффективности вида $k \in N_{s}$ инвестиционного проекта $j \in N_{n}$ в случае, когда рынок находится в состоянии $i \in N_{m}$. В этом контексте исходными данными задачи является трехмерная матрица эффективности проектов $E$ размера $m \times n \times s$ с элементами $e_{i j k}$ из $\mathbf{R}$.

На множестве инвестиционных портфелей $X$ зададим векторную целевую функцию

$$
f(x, E)=\left(f_{1}\left(x, E_{1}\right), f_{2}\left(x, E_{2}\right), \ldots, f_{s}\left(x, E_{s}\right)\right),
$$

компонентами которой являются максиминные критерии Вальда [7]

$$
f_{k}\left(x, E_{k}\right)=\min _{i \in N_{m}} E_{i k} x=\min _{i \in N_{m}} \sum_{j \in N_{n}} e_{i j k} x_{j} \rightarrow \max _{x \in X}, \quad k \in N_{s},
$$

где $E_{k} \in \mathbf{R}^{m \times n}-k$-е сечение матрицы $E=\left[e_{i j k}\right] \in \mathbf{R}^{m \times n \times s}, E_{i k}=\left(e_{i 1 k}, e_{i 2 k}, \ldots, e_{i n k}\right)$ - $i$-я строка этого сечения. Таким образом, следуя критерию Вальда, инвестор в условиях нестабильности экономической ситуации проявляет крайнюю осторожность, оптимизируя эффективность портфеля $E_{i k} x$ в предположении, что рынок находится в самом невыгодном для него состоянии.

Под векторной (s-критериальной) инвестиционной булевой задачей $Z^{s}(E), s \geqslant 1$, будем понимать задачу поиска множества Парето, состоящего из парето-оптимальных инвестиционных портфелей

$$
P^{s}(E)=\left\{x \in X: P^{s}(x, E)=\varnothing\right\}
$$

где

$$
\begin{aligned}
& P^{s}(x, E)=\left\{x^{\prime} \in X: x^{\prime} \succ x\right\}, \\
& x^{\prime} \succsim x \Longleftrightarrow g\left(x^{\prime}, x, E\right) \geqslant \mathbf{0}^{(s)} \& g\left(x^{\prime}, x, E\right) \neq \mathbf{0}^{(s)}, \\
& g\left(x^{\prime}, x, E\right)=\left(g_{1}\left(x^{\prime}, x, E_{1}\right), g_{2}\left(x^{\prime}, x, E_{2}\right), \ldots, g_{s}\left(x^{\prime}, x, E_{s}\right)\right)^{T}, \\
& g_{k}\left(x^{\prime}, x, E_{k}\right)=f_{k}\left(x^{\prime}, E_{k}\right)-f_{k}\left(x, E_{k}\right) \\
&=\max _{i \in N_{m}} \min _{i^{\prime} \in N_{m}}\left(E_{i^{\prime} k} x^{\prime}-E_{i k} x\right), \quad k \in N_{s}, \\
& \mathbf{0}^{(s)}=(0,0, \ldots, 0)^{T} \in \mathbf{R}^{s} .
\end{aligned}
$$

Легко также видеть, что в частном случае при $m=1$ наша задача $Z^{s}(E)$ превращается в векторную булеву задачу с линейными критериями (на максимум). Такой случай можно интерпретировать, как ситуацию, при которой состояние рынка не вызывает сомнений.

В пространстве портфелей $\mathbf{R}^{n}$ зададим линейную норму $l_{1}$, а в пространстве состояний 
рынка $\mathbf{R}^{m}$ и критериальном пространстве эффективности $\mathbf{R}^{s}-$ чебышевскую норму $l_{\infty}$ :

$$
\begin{array}{rlrl}
\left\|E_{i k}\right\|_{1} & =\sum_{j \in N_{n}}\left|e_{i j k}\right|, & i \in N_{m}, \quad k \in N_{s}, \\
\left\|E_{k}\right\|_{\infty} & =\max _{i \in N_{m}}\left\|E_{i k}\right\|_{1}=\max _{i \in N_{m}} \sum_{j \in N_{n}}\left|e_{i j k}\right|, \quad k \in N_{s}, \\
\|E\|_{\infty} & =\max _{k \in N_{s}}\left\|E_{k}\right\|_{\infty}=\max _{k \in N_{s}} \max _{i \in N_{m}}\left\|E_{i k}\right\|_{1} & \\
& =\max _{k \in N_{s}} \max _{i \in N_{m}} \sum_{j \in N_{n}}\left|e_{i j k}\right| .
\end{array}
$$

Таким образом, для любых индексов $i \in N_{m}$ и $k \in N_{s}$ верны неравенства

$$
\left\|E_{i k}\right\|_{1} \leqslant\left\|E_{k}\right\|_{\infty} \leqslant\|E\|_{\infty} .
$$

Кроме того, для всякого сечения $E_{k} \in \mathbf{R}^{m \times n}$ и любых портфелей $x, x^{\prime} \in X$ справедливы неравенства

$$
\begin{aligned}
E_{i k} x-E_{i^{\prime} k} x^{\prime} & \geqslant-\left\|E_{i k}\right\|_{1}-\left\|E_{i^{\prime} k}\right\|_{1} \\
& \geqslant-2\left\|E_{k}\right\|_{\infty}, \quad i, i^{\prime} \in N_{m}, \quad k \in N_{s} .
\end{aligned}
$$

Следуя $[8,9,10]$, радиусом устойчивости задачи $Z^{s}(E), s \geqslant 1$, назовем число

$$
\rho=\rho(m, n, s)= \begin{cases}\sup \Xi & \text { если } \Xi \neq \varnothing, \\ 0 & \text { если } \Xi=\varnothing,\end{cases}
$$

где

$$
\Xi=\left\{\varepsilon>0: P^{s}\left(E+E^{\prime}\right) \subseteq P^{s}(E) \text { для всех } E^{\prime} \in \Omega(\varepsilon)\right\}
$$

и

$$
\Omega(\varepsilon)=\left\{E^{\prime} \in \mathbf{R}^{m \times n \times s}:\left\|E^{\prime}\right\|_{\infty}<\varepsilon\right\}
$$

- множество возмущающих матриц, $P^{s}\left(E+E^{\prime}\right)$ - множество Парето возмущенной задачи $Z^{s}\left(E+E^{\prime}\right)$. Тем самым, радиус устойчивости задачи задает предельный уровень возмущений элементов матрицы $E$, не приводящих к появлению новых парето-оптимальных портфелей.

Ясно, что при выполнении равенства

$$
P^{s}(E)=X
$$

радиус устойчивости задачи $Z^{s}(E)$ равен бесконечности. Поэтому в дальнейшем этот случай будем исключать из рассмотрения, а задачу $Z^{s}(E)$, для которой множество $X \backslash P^{s}(E)$ непусто, будем называть нетривиальной.

\section{3. Оценки радиуса устойчивости}

Для нетривиальной задачи $Z^{s}(E)$ положим

$$
\varphi=\varphi(m, n, s)=\min _{x \notin P^{s}(E)} \max _{x^{\prime} \in P^{s}(x, E)} \min _{k \in N_{s}} \max _{i \in N_{m}} \min _{i^{\prime} \in N_{m}}\left(E_{i^{\prime} k} x^{\prime}-E_{i k} x\right) .
$$


Поскольку для любого портфеля $x \notin P^{s}(E)$ множество $P^{s}(x, E)$ непусто, то формула

$$
x^{\prime} \underset{E}{\succ} x
$$

верна для всех $x \notin P^{s}(E)$ и $x^{\prime} \in P^{s}(x, E)$. Поэтому $\varphi \geqslant 0$.

Теорема 1. Для радиуса устойчивости $\rho(m, n, s)$ векторной нетривиальной инвестищионной задачи $Z^{s}(E), s \geqslant 1$, справедливы оценки

$$
\frac{1}{2} \varphi(m, n, s) \leqslant \rho(m, n, s) \leqslant n \varphi(m, n, s) .
$$

Доказательство. Для доказательства теоремы 1 сначала убедимся в справедливости неравенства $\rho \geqslant \varphi / 2$. Это неравенство очевидно, если $\varphi=0$. Пусть $\varphi>0$. Согласно определению числа $\varphi$, для любого портфеля $x \notin P^{s}(E)$ существует такой портфель $x^{0} \in P^{s}(x, E)$, что

$$
\max _{i \in N_{m}} \min _{i^{\prime} \in N_{m}}\left(E_{i^{\prime} k} x^{0}-E_{i k} x\right) \geqslant \varphi, \quad k \in N_{s} .
$$

Отсюда, учитывая (1), для любой матрицы $E^{\prime} \in \mathbf{R}^{m \times n \times s}$ и всякого индекса $k \in N_{s}$ получаем

$$
\begin{aligned}
g_{k}\left(x^{0}, x, E_{k}+E_{k}^{\prime}\right) & =\max _{i \in N_{m}} \min _{i^{\prime} \in N_{m}}\left(E_{i^{\prime} k} x^{0}-E_{i k} x+E_{i^{\prime} k}^{\prime} x^{0}-E_{i k}^{\prime} x\right) \\
& \geqslant \max _{i \in N_{m}} \min _{i^{\prime} \in N_{m}}\left(E_{i^{\prime} k} x^{0}-E_{i k} x\right)-2\left\|E_{k}^{\prime}\right\|_{\infty} \\
& \geqslant \varphi-2\left\|E_{k}^{\prime}\right\|_{\infty} .
\end{aligned}
$$

Поэтому, полагая $E^{\prime} \in \Omega(\varphi / 2)$, т.е., $\left\|E_{k}^{\prime}\right\|_{\infty}<\varphi / 2, k \in N_{s}$, получаем что

$$
g_{k}\left(x^{0}, x, E_{k}+E_{k}^{\prime}\right)>0, \quad k \in N_{s} ;
$$

это означает, что $x^{0} \underset{E+E^{\prime}}{\succ} x$, т.е. $x \notin P^{s}\left(E+E^{\prime}\right)$. Резюмируя и учитывая, что $x \notin P^{s}(E)$, заключаем, что $P^{s}\left(E+E^{\prime}\right) \subseteq P^{s}(E)$ для всех $E^{\prime} \in \Omega(\varphi / 2)$. Следовательно, справедливо неравенство

$$
\rho(m, n, s) \geqslant \varphi(m, n, s) / 2 \text {. }
$$

Далее, докажем неравенство $\rho \leqslant n \varphi$. Согласно определению числа $\varphi$, существует такой портфель $x^{*} \notin P^{s}(E)$, что для любого портфеля $x \in P^{s}\left(x^{*}, E\right)$ найдется индекс $l=l(x) \in N_{s}$, удовлетворяющий условию

$$
\max _{i \in N_{m}} \min _{i^{\prime} \in N_{m}}\left(E_{i^{\prime} l} x-E_{i l} x^{*}\right) \leqslant \varphi .
$$

Полагая $\varepsilon>n \varphi$, рассмотрим возмущающую матрицу $E^{0}=\left[e_{i j k}^{0}\right] \in \mathbf{R}^{m \times n \times s}$, элементы которой определим следующим образом:

$$
e_{i j k}^{0}= \begin{cases}\delta & \text { если } i \in N_{m}, x_{j}^{*}=1, k \in N_{s}, \\ -\delta & \text { в противном случае },\end{cases}
$$

где

$$
\varphi<\delta<\frac{\varepsilon}{n}
$$


Тогда справедливы равенства

$$
\left\|E^{0}\right\|_{\infty}=\left\|E_{k}^{0}\right\|_{\infty}=\left\|E_{i k}^{0}\right\|_{1}=n \delta, \quad i \in N_{m}, \quad k \in N_{s} .
$$

Поэтому $E^{0} \in \Omega(\varepsilon)$. Кроме того, все строки $E_{i k}^{0}, i \in N_{m}$, любого сечения $E_{k}^{0}, k \in N_{s}$, одинаковы и состоят из компонент $\delta$ и $-\delta$. Обозначив такую строку через $A$, получаем, что

$$
A\left(x-x^{*}\right)=-\delta\left\|x-x^{*}\right\|_{1} \leqslant-\delta<-\varphi \leqslant 0 .
$$

Отсюда, учитывая (2) и строение возмущающей матрицы $E^{0}$, выводим, что для любого портфеля $x \in P^{s}\left(x^{*}, E\right)$ справедливы соотношения

$$
\begin{aligned}
g_{l}\left(x, x^{*}, E_{l}+E_{l}^{0}\right) & =\min _{i \in N_{m}}\left(E_{i l}+A\right) x-\min _{i \in N_{m}}\left(E_{i l}+A\right) x^{*} \\
& =\max _{i \in N_{m}} \min _{i^{\prime} \in N_{m}}\left(E_{i^{\prime} l} x-E_{i l} x^{*}\right)+A\left(x-x^{*}\right)<0 .
\end{aligned}
$$

В результате получаем, что для любого $x \in P^{s}\left(x^{*}, E\right)$

$$
x \notin P^{s}\left(x^{*}, E+E^{0}\right) .
$$

Допустим теперь, что портфель $x \notin P^{s}\left(x^{*}, E\right)$. Тогда возможны лишь следующие два случая.

Случай 1: $g\left(x, x^{*}, E\right)=\mathbf{0}^{(s)}$. Тогда для любого $k \in N_{s}$ соотношения (3) влекут

$$
\begin{aligned}
g_{k}\left(x, x^{*}, E_{k}+E_{k}^{0}\right) & =\min _{i \in N_{m}}\left(E_{i k}+A\right) x-\min _{i \in N_{m}}\left(E_{i k}+A\right) x^{*} \\
& =g_{k}\left(x, x^{*}, E_{k}\right)+A\left(x-x^{*}\right)<0 .
\end{aligned}
$$

Case 2. Существует такой индекс $p \in N_{s}$, что $g_{p}\left(x, x^{*}, E_{p}\right)<0$. Тогда, вновь используя (3), получаем, что

$$
g_{p}\left(x, x^{*}, E_{p}+E_{p}^{0}\right)<0 .
$$

Таким образом, $x \notin P^{s}\left(x^{*}, E+E^{0}\right)$, если $x \notin P^{s}\left(x^{*}, E\right)$. Этот факт вместе с (4) дает $P^{s}\left(x^{*}, E+E^{0}\right)=\varnothing$, т.е. $x^{*}$ является парето-оптимальным портфелем возмущенной задачи $Z^{s}\left(E+E^{0}\right)$. Отсюда, так как $x^{*} \notin P^{s}(E)$, заключаем, что для любого $\varepsilon>n \varphi$ найдется такое $E^{0} \in \Omega(\varepsilon)$, что $P^{s}\left(E+E^{0}\right) \nsubseteq P^{s}(E)$. Следовательно, справедливо неравенство

$$
\rho(m, n, s) \leqslant n \varphi(m, n, s) .
$$

Теорема 1 доказана.

Следствие 1. Радиус устойчивости $\rho(m, n, s)$ равен нулю тогда и только тогда, когда $\varphi(m, n, s)=0$.

\section{4. Достижимость нижней оценки}

Покажем, что нижняя оценка радиуса устойчивости задачи, указанная теоремой 1 , является достижимой. 
Теорема 2. При $m \geqslant 2$ и $n \geqslant 2$ существует такой класс векторных инвестиционных задач $Z^{s}(E), s \geqslant 1$, что для радиуса устойчивости любой задачи этого класса справедлива формула

$$
\rho(m, n, s)=\varphi(m, n, s) / 2 .
$$

Доказательство. Для доказательства равенства $\rho=\varphi / 2$ согласно теореме 1 достаточно выделить класс задач, для которых $\rho \leqslant \varphi / 2$. Дальнейшее изложение и посвятим этому, считая, что $\varphi>0$.

Будем рассматривать класс задач $Z^{s}(E)$ с условиями

$$
X=\left\{x^{0}, x^{*}\right\}, \quad P^{s}\left(x^{*}, E\right)=\left\{x^{0}\right\},
$$

то есть

$$
x^{0} \underset{E}{\succ} x^{*}, \quad x^{*} \notin P^{s}(E) .
$$

Тогда существует такой индекс $l \in N_{s}$, что

$$
g_{l}\left(x^{0}, x^{*}, E_{l}\right)=\varphi>0 .
$$

Будем также считать, что

$$
\left(E_{i\left(x^{0}\right) l}-E_{i\left(x^{*}\right) l}\right) x^{*}>\varphi / 2,
$$

где

$$
\begin{aligned}
& i\left(x^{0}\right)=\arg \min \left\{E_{i l} x^{0}: i \in N_{m}\right\}, \\
& i\left(x^{*}\right)=\arg \min \left\{E_{i l} x^{*}: i \in N_{m}\right\} .
\end{aligned}
$$

Тогда очевидно, что $i\left(x^{0}\right) \neq i\left(x^{*}\right)$, поскольку $\varphi>0$.

Далее будем предполагать, что существуют таких два индекса $p \neq q$ множества $N_{n}$, что $x_{p}^{0}=x_{q}^{*}=1, x_{q}^{0}=x_{p}^{*}=0$.

Для любого числа $\varepsilon>\varphi / 2$ элементы возмущающей матрицы $E^{0}=\left[e_{i j k}^{0}\right] \in \mathbf{R}^{m \times n \times s}$ зададим по правилу

$$
e_{i j k}^{0}= \begin{cases}-\delta & \text { если } i=i\left(x^{0}\right), j=p, k=l, \\ \delta & \text { если } i \in N_{m} \backslash\left\{i\left(x^{0}\right)\right\}, j=q, k=l, \\ 0 & \text { в противном случае, }\end{cases}
$$

где

$$
\frac{1}{2} \varphi<\delta<\min \left\{\varepsilon,\left(E_{i\left(x^{0}\right) l}-E_{i\left(x^{*}\right) l}\right) x^{*}\right\} .
$$

Отметим, что последние неравенства корректны благодаря (8). Согласно построенной возмущающей матрице $E^{0}$, очевидны следующие равенства:

$$
\begin{aligned}
E_{i\left(x^{0}\right) l}^{0} x^{0} & =-\delta, & & \\
E_{i l}^{0} x^{0} & =0, & & i \in N_{m} \backslash\left\{i\left(x^{0}\right)\right\}, \\
E_{i l}^{0} x^{*} & =\delta, & & i \in N_{m} \backslash\left\{i\left(x^{0}\right)\right\}, \\
E_{i\left(x^{0}\right) l}^{0} x^{*} & =0, & & \\
\left\|E_{i l}^{0}\right\|_{1}=\left\|E_{l}^{0}\right\|_{\infty}=\left\|E^{0}\right\|_{\infty} & =\delta, & & i \in N_{m}, \quad E^{0} \in \Omega(\varepsilon)
\end{aligned}
$$


Далее докажем, что инвестиционный портфель $x^{*} \in P^{s}\left(E+E^{0}\right)$. Для этого в силу (6) достаточно убедиться, что $x^{0} \notin P^{s}\left(x^{*}, E+E^{0}\right)$. Согласно (11) и (12) имеем

$$
\begin{aligned}
f_{l}\left(x^{0}, E_{l}+E_{l}^{*}\right) & =\min \left\{\left(E_{i\left(x^{0}\right) l}+E_{i\left(x^{0}\right) l}^{0}\right) x^{0}, \min _{i \neq i\left(x^{0}\right)}\left(E_{i l}+E_{i l}^{0}\right) x^{\}}\right. \\
& =\min \left\{f_{l}\left(x^{0}, E_{l}\right)-\delta, \min _{i \neq i\left(x^{0}\right)} E_{i l} x^{0}\right\} \\
& =f_{l}\left(x^{0}, E_{l}\right)-\delta .
\end{aligned}
$$

Теперь докажем равенство

$$
f_{l}\left(x^{*}, E_{l}+E_{l}^{*}\right)=f_{l}\left(x^{*}, E_{l}\right)+\delta .
$$

Из равенств (13) и неравенства $i\left(x^{0}\right) \neq i\left(x^{*}\right)$ следует, что

$$
\begin{aligned}
f_{l}\left(x^{*}, E_{l}+E_{l}^{*}\right) & =\min \left\{\left(E_{i\left(x^{*}\right) l}+E_{i\left(x^{*}\right) l}^{0}\right) x^{*}, \min _{i \neq i\left(x^{*}\right)}\left(E_{i l}+E_{i l}^{0}\right) x^{*}\right\} \\
& =\min \left\{f_{l}\left(x^{*}, E_{l}\right)+\delta, \min _{i \neq i\left(x^{*}\right)}\left(E_{i l}+E_{i l}^{0}\right) x^{*}\right\} .
\end{aligned}
$$

Поэтому, с учетом очевидных ввиду (13) соотношений

$$
f_{l}\left(x^{*}, E_{l}\right)+\delta=\min _{i \in N_{m}} E_{i l} x^{*}+\delta \leqslant\left(E_{i l}+E_{i l}^{0}\right) x^{*}, \quad i \in N_{m} \backslash\left\{i\left(x^{0}\right)\right\},
$$

для доказательства равенства (16) остается убедиться, что

$$
f_{l}\left(x^{*}, E_{l}\right)+\delta \leqslant\left(E_{i\left(x^{0}\right) l}+E_{i\left(x^{0}\right) l}^{0}\right) x^{*} .
$$

Для этого, воспользовавшись (10) и (14), выводим

$$
f_{l}\left(x^{*}, E_{l}\right)+\delta-\left(E_{i\left(x^{0}\right) l}+E_{i\left(x^{0}\right) l}^{0}\right) x^{*}=\delta-\left(E_{i\left(x^{0}\right) l}-E_{i\left(x^{*}\right) l}\right) x^{*}<0 .
$$

Итак, равенство (16) доказано. Наконец, последовательно применяя (15), (16), (7) и (10), получаем, что

$$
\begin{aligned}
g_{l}\left(x^{0}, x^{*}, E_{l}+E_{l}^{0}\right) & =f_{l}\left(x^{0}, E_{l}+E_{l}^{0}\right)-f_{l}\left(x^{*}, E_{l}+E_{l}^{0}\right) \\
& =g_{l}\left(x^{0}, x^{*}, E_{l}\right)-2 \delta=\varphi-2 \delta<0,
\end{aligned}
$$

т.е. $x^{0} \notin P^{s}\left(x^{*}, E+E^{0}\right)$. Это и доказывает, что $x^{*}$ является парето-оптимальным инвестиционным портфелем возмущенной задачи $Z^{s}\left(E+E^{0}\right)$. Отсюда, поскольку $x^{*} \notin P^{s}(E)$, заключаем, что для любого $\varepsilon>\varphi / 2$ найдется такое $E^{0} \in \Omega(\varepsilon)$, что $P^{s}\left(E+E^{0}\right) \nsubseteq P^{s}(E)$. Поэтому справедливо неравенство

$$
\rho(m, n, s) \leqslant \varphi(m, n, s) / 2 .
$$

Следовательно, в силу теоремы 1 формула (5) верна. Теорема 2 доказана.

Приведем числовой пример, иллюстрирующий теорему 2.

Пример 1. Пусть $m=3, n=3, s=1 ; X=\left\{x^{0}, x^{*}\right\}, x^{0}=(0,1,1)^{T}, x^{*}=(1,1,0)^{T}$;

$$
E=\left(\begin{array}{ccc}
1 & -1 & 2 \\
-3 & 0 & 4 \\
1 & -1 & 2
\end{array}\right)
$$


- матрица со строками $E_{i}, i \in N_{3}$. Тогда

$$
f\left(x^{0}, E\right)=1, \quad f\left(x^{*}, E\right)=-3, \quad i\left(x^{0}\right)=1, \quad i\left(x^{*}\right)=2 .
$$

Отсюда следует, что $x^{0} \in P^{1}(E), x^{*} \notin P^{1}(E)$. Поэтому

$$
\varphi=g\left(x^{0}, x^{*}, E\right)=4 .
$$

Покажем, что радиус устойчивости

$$
\rho(3,3,1) \leqslant \varphi / 2=2 \text {. }
$$

Легко видеть, что неравенство (8) принимает вид

$$
\left(E_{1}-E_{2}\right) x^{*}=3>2=\varphi / 2,
$$

а возмущающая матрица $E^{0}$ со строками $E_{i}^{0}, i \in N_{3}$, построенная по правилу (9), представляется в виде

$$
E^{0}=\left(\begin{array}{ccc}
0 & 0 & -\delta \\
\delta & 0 & 0 \\
\delta & 0 & 0
\end{array}\right), \quad 2<\delta<3
$$

Поэтому, учитывая равенство

$$
E+E^{0}=\left(\begin{array}{ccc}
1 & -1 & 2-\delta \\
-3+\delta & 0 & 4 \\
1+\delta & -1 & 2
\end{array}\right)
$$

получаем, что

$$
\begin{aligned}
f\left(x^{0}, E+E^{0}\right) & =\min _{i \in N_{3}}\left(E_{i}+E_{i}^{0}\right) x^{0} \\
& =\min \{1-\delta, 4,1\}=1-\delta, \\
f\left(x^{*}, E+E^{0}\right) & =\min _{i \in N_{3}}\left(E_{i}+E_{i}^{0}\right) x^{*} \\
& =\min \{0,-3+\delta, \delta\}=-3+\delta .
\end{aligned}
$$

Отсюда, на основании неравенств $2<\delta<3$, заключаем, что

$$
g\left(x^{0}, x^{*}, E+E^{0}\right)=4-2 \delta<0,
$$

то есть $x^{*} \in P^{1}\left(E+E^{0}\right)$. Это включение вместе с соотношениями

$$
\left\|E^{0}\right\|_{\infty}=\delta>2
$$

$x^{*} \notin P^{1}(E)$ дает

$$
\rho=\rho(3,3,1) \leqslant 2 .
$$

Следовательно, учитывая теорему 1, заключаем, что

$$
\rho(3,3,1)=\varphi / 2=2 .
$$




\section{5. Достижимость верхней оценки}

Покажем, что верхняя оценка $n \varphi(m, n, s)$ радиуса устойчивости задачи $Z^{s}(E)$ достигается при $m=s=1$.

Теорема 3. При $m=s=1$ существует такой класс скалярных инвестищионных задач $Z^{1}(E), E \in \mathbf{R}^{1 \times n \times 1}$, что для радиуса устойчивости любой задачи этого класса справедлива формула

$$
\rho(1, n, 1)=n \varphi(1, n, 1) .
$$

Доказательство. В силу теоремы 1, для доказательства равенства

$$
\rho(1, n, 1)=n \varphi(1, n, 1)
$$

достаточно указать класс задач с условием

$$
\rho(1, n, 1) \geqslant n \varphi(1, n, 1),
$$

где $\varphi(1, n, 1)>0$.

Покажем, что такой класс существует при $X=\left\{x^{*}, x^{1}, x^{2}, \ldots, x^{n}\right\} \subset \mathbf{E}^{n}, n \geqslant 2$, где $x^{*}=\mathbf{0}^{(n)}, x^{j}=e^{j}, j \in N_{n}$. Здесь $e^{j}-$ единичный вектор-столбец пространства $\mathbf{R}^{n}$, иными словами, $e^{j}$ есть $j$-й столбец единичной матрицы размера $n \times n$. Поскольку $m=s=1$, положим $E=(a, a, \ldots, a) \in \mathbf{R}^{n}$, где $a>0$. Поэтому $f\left(x^{*}, E\right)=0$, $f\left(x^{j}, E\right)=a, j \in N_{n}$, то есть $x^{*} \notin P^{1}(E), x^{j} \in P^{1}(E)=P^{1}\left(x^{*}, E\right), j \in N_{n}$. Отсюда, согласно определению числа $\varphi(1, n, 1)$, справедливо равенство

$$
\varphi=\varphi(1, n, 1)=a \text {. }
$$

Пусть теперь $E^{\prime}=\left(e_{1}^{\prime}, e_{2}^{\prime}, \ldots, e_{n}^{\prime}\right)-$ произвольная возмущающая вектор-строка из множества строк $\Omega(n a)$, то есть

$$
\left\|E^{\prime}\right\|_{1}=\sum_{j \in N_{n}}\left|e_{j}^{\prime}\right|<n a .
$$

Методом от противного легко доказать, что существует хотя бы один такой индекс $p$, что $\left|e_{p}^{\prime}\right|<a$. Поэтому

$$
g\left(x^{p}, x^{*}, E+E^{\prime}\right)=\left(E+E^{\prime}\right)\left(x^{p}-x^{*}\right)=a+e_{p}^{\prime}>0 .
$$

Отсюда заключаем, что при любой возмущающей строке $E^{\prime} \in \Omega(n \varphi)$ портфель $x^{*}$ не принадлежит $P^{1}\left(E+E^{\prime}\right)$. Следовательно, так как $x^{*} \notin P^{1}(E)$, получаем, что

$$
\rho(1, n, 1) \geqslant n \varphi(1, n, 1) .
$$

Теорема 3 доказана.

\section{6. Условия устойчивости задачи}

Векторную инвестиционную задачу $Z^{s}(E), s \geqslant 1$, назовем устойчивой, если ее радиус устойчивости больше нуля. Введем в рассмотрение множество Слейтера (см. [11])

$$
S l^{S}(E)=\left\{x \in X: S l^{S}(x, E)=\varnothing\right\},
$$


где

$$
S l^{S}(x, E)=\left\{x^{\prime} \in X: g_{k}\left(x^{\prime}, x, E_{k}\right)>0 \text { для всех } k \in N_{s}\right\} .
$$

Очевидно, что $P^{s}(E) \subseteq S l^{s}(E)$ при любой матрице $E \in \mathbf{R}^{m \times n \times s}$.

Теорема 4. Для векторной нетривиальной инвестиционной задачи $Z^{s}(E), s \geqslant 1$, следующие утверждения эквивалентны:

(i) задача $Z^{s}(E)$ устойчива,

(ii) $P^{s}(E)=S l^{s}(E)$,

(iii) $\varphi(m, n, s)>0$.

Доказательство. Вначале докажем, что (i) $\Rightarrow$ (ii). Предположим, что задача $Z^{s}(E)$ устойчива, но $P^{s}(E) \neq S l^{S}(E)$. Тогда существует инвестиционный портфель $x^{*} \in S l^{S}(E) \backslash$ $P^{s}(E)$. Поэтому $S l^{s}\left(x^{*}, E\right)=\varnothing$ и $P^{s}\left(x^{*}, E\right) \neq \varnothing$. Это означает, что найдется такое $x^{*} \notin P^{s}(E)$, что для любого $x \in P^{s}\left(x^{*}, E\right)$ существует такой индекс $l \in N_{s}$, что $g_{l}\left(x, x^{*}, E_{l}\right)=0$. Следовательно,

$$
\varphi(m, n, s)=0,
$$

и, согласно следствию 1 ,

$$
\rho(m, n, s)=0
$$

что противоречит устойчивости задачи $Z^{s}(E)$.

Докажем, что (ii) $\Rightarrow$ (iii). Если $P^{s}(E)=S l^{s}(E)$, то для любого портфеля $x \notin P^{s}(E)$ множество $S l^{s}(x, E)$ непусто. Поэтому существует такой портфель $x^{0} \in X$, что верны неравенства $g_{k}\left(x^{0}, x, E_{k}\right)>0, k \in N_{s}$, иными словами, $x^{0} \in P^{s}(x, E)$. Таким образом, для любого $x \notin P^{s}(E)$ найдется такое $x^{0} \in P^{s}(x, E)$, что для любого индекса $k \in N_{s}$ справедливо неравенство

$$
g_{k}\left(x^{0}, x, E_{k}\right)>0 .
$$

Следовательно,

$$
\varphi(m, n, s)>0 \text {. }
$$

Импликация (iii) $\Rightarrow$ (i) очевидна в силу теоремы 1 .

Теорема 4 доказана.

Поскольку $P^{1}(E)=S l^{1}(E)$, то из теоремы 4 вытекает следующее утверждение.

Следствие 2. Скалярная инвестищионная задача $Z^{1}(E)$ устойчива при любой матрице $E \in \mathbf{R}^{m \times n}$. 


\section{7. Случай линейных критериев $(m=1)$}

При $m=1$ наша векторная инвестиционная задача превращается в векторную задачу линейного булева программирования:

$$
f_{k}\left(x, E_{k}\right)=E_{k} x \rightarrow \max _{x \in X}, \quad k \in N_{s},
$$

где $E_{k} \in \mathbf{R}^{1 \times n}, k \in N_{s},-k$-я строка матрицы $E=\left[e_{1 j k}\right] \in \mathbf{R}^{1 \times n \times s}, X \subseteq \mathbf{E}^{n}$, при этом, как и ранее, в критериальном пространстве $\mathbf{R}^{s}$ задана норма $l_{\infty}$, а в пространстве решений $\mathbf{R}^{n}$ - норма $l_{1}$.

Теорема 5. Для радиуса устойчивости $\rho(1, n, s), s \geqslant 1$, векторной нетривиальной задачи линейного булева программирования (17) справедливы достижимые оченки

$$
\varphi(1, n, s) \leqslant \rho(1, n, s) \leqslant n \varphi(1, n, s) .
$$

Доказательство. Прежде всего отметим, что справедливость и достижимость верхней оценки $n \varphi(1, n, s)$ следуют из теорем 1 и 3.

Далее покажем, что

$$
\rho(1, n, s) \geqslant \varphi(1, n, s) .
$$

Как обычно, будем полагать, что

$$
\varphi=\varphi(1, n, s)>0 .
$$

Исходя из определения $\varphi$, легко убеждаемся, что для любого $x \notin P^{s}(E)$ найдется такое $x^{0} \in P^{s}(x, E)$, что для любого индекса $k \in N_{s}$ выполняется соотношение

$$
E_{k}\left(x^{0}-x\right) \geqslant \varphi .
$$

Теперь, полагая $E^{\prime} \in \Omega(\varphi)$, откуда следует, что $\left\|E_{k}^{\prime}\right\|_{1}<\varphi, k \in N_{s}$, получаем, что

$$
\left(E_{k}+E_{k}^{\prime}\right)\left(x^{0}-x\right) \geqslant E_{k}\left(x^{0}-x\right)-\left\|E_{k}^{\prime}\right\|_{1} \geqslant \varphi-\left\|E_{k}^{\prime}\right\|_{1}>0, \quad k \in N_{s} .
$$

Это значит, что $x \notin P^{s}\left(E+E^{\prime}\right)$. Итак, для любой возмущающей матрицы $E^{\prime} \in \Omega(\varphi)$ верно включение $P^{s}\left(E+E^{\prime}\right) \subseteq P^{s}(E)$. Следовательно, справедлива нижняя оценка радиуса устойчивости (см. (18)).

Наконец, докажем достижимость нижней оценки. Для этого построим класс задач, для которых

$$
\rho(1, n, s) \leqslant \varphi=\varphi(1, n, s)
$$

при $\varphi>0$.

Пусть

$$
X=\left\{x^{0}, x^{*}\right\}, \quad P^{s}\left(x^{*}, E\right)=\left\{x^{0}\right\},
$$

$E \in \mathbf{R}^{1 \times n \times s}$, и пусть существует такой индекс $p \in N_{n}$, что $x_{p}^{0}=1, x_{p}^{*}=0$. Тогда, так как $x^{*} \notin P^{s}(E)$, в силу определения $\varphi$ найдется такой индекс $l \in N_{s}$, что

$$
E_{l}\left(x^{0}-x^{*}\right)=\varphi>0 .
$$


Полагая $\varphi<\delta<\varepsilon$, построим возмущающую матрицу $E^{0}=\left[e_{1 j k}^{0}\right] \in \mathbf{R}^{1 \times n \times s}$, элементы которой зададим по правилу

$$
e_{1 j k}^{0}= \begin{cases}-\delta & \text { если } j=p, k=l, \\ 0 & \text { в противном случае. }\end{cases}
$$

Тогда получаем, что

$$
\begin{aligned}
\left\|E^{0}\right\|_{\infty}=\left\|E_{l}^{0}\right\|_{1} & =\delta, \\
E_{l}^{0}\left(x^{0}-x^{*}\right) & =-\delta .
\end{aligned}
$$

Отсюда и из (19) получаем, что

$$
\begin{aligned}
g_{l}\left(x^{0}, x^{*}, E_{l}+E_{l}^{0}\right) & =\left(E_{l}+E_{l}^{0}\right)\left(x^{0}-x^{*}\right) \\
& =\varphi-\delta<0 .
\end{aligned}
$$

Поэтому $x^{0} \notin P^{s}\left(x^{*}, E+E^{0}\right)$, то есть $x^{*} \in P^{s}\left(E+E^{0}\right)$, а поскольку $x^{*} \notin P^{s}(E)$, для произвольного $\varepsilon>\varphi$ найдется такое $E^{0} \in \Omega(\varepsilon)$, что $\left.P^{s}\left(E+E^{0}\right) \nsubseteq P^{s}(E)\right)$, откуда следует оценка

$$
\rho(1, n, s) \leqslant \varphi(1, n, s)
$$

Следовательно, согласно доказанному выше соотношению (18), нижняя оценка $\varphi(1, n, s)$ достижима. Теорема 5 доказана.

Приведем числовой пример, свидетельствующий о достижимости нижней оценки радиуса устойчивости, указанной теоремой 5.

Пример 2. Пусть $m=1, n=3, s=2 ; X=\left\{x^{0}, x^{*}\right\}, x^{0}=(0,1,1)^{T}, x^{*}=(1,1,0)^{T}$;

$$
E=\left(\begin{array}{lll}
1 & -1 & 2 \\
1 & -1 & 2
\end{array}\right)
$$

Тогда

$$
p=3, \quad f\left(x^{0}, E\right)=(1,1), \quad f\left(x^{*}, E\right)=(0,0),
$$

иными словами,

$$
\left\{x^{0}\right\}=P^{2}\left(x^{*}, E\right),
$$

$x^{*} \notin P^{2}(E)$. Отсюда (см. (19))

$$
\varphi=\varphi(1,3,2)=E_{1}\left(x^{0}-x^{*}\right)=1,
$$

то есть $l=1$. Поэтому элементы возмущающей матрицы $E^{0}$, вычисленные по формуле (20), имеют вид

$$
\begin{aligned}
& e_{1 p l}^{0}=e_{131}^{0}=-\delta, \\
& e_{1 j k}=0, \quad(j, k) \neq(3,1),
\end{aligned}
$$


где

$$
1=\varphi<\delta<\varepsilon \text {. }
$$

В результате, поскольку $E_{1}^{0}=(0,0,-\delta)$, получаем, что

$$
g_{1}\left(x^{0}, x^{*}, E_{1}+E_{1}^{0}\right)=\left(E_{1}+E_{1}^{0}\right)\left(x^{0}-x^{*}\right)=1-\delta<0 .
$$

Это означает, что $x^{0} \notin P^{2}\left(x^{*}, E+E^{0}\right)$, а поэтому $x^{*} \in P^{2}\left(E+E^{0}\right)$. Последнее включение вместе с соотношениями

$$
\left\|E^{0}\right\|_{\infty}=\delta>\varphi
$$

и $x^{*} \notin P^{2}(E)$ дает

$$
\rho(1,3,2) \leqslant \varphi(1,3,2)=1 \text {. }
$$

Следовательно, согласно теореме 5,

$$
\rho(1,3,2)=1 \text {. }
$$

В заключение отметим, что ранее в [12] были получены нижняя и верхняя оценки радиуса устойчивости векторной ( $s$-критериальной) задачи линейного булева программирования (17) при условии, что

$$
X=\left\{x \in \mathbf{E}^{n}: A x \leqslant b\right\},
$$

а возмущениям повергались все параметры задачи, то есть не только элементы матрицы $E$, но и элементы матрицы $A \in \mathbf{R}^{q \times n}$ и вектора $b \in \mathbf{R}^{q}$, при этом во всех пространствах варьирующих параметров $\mathbf{R}^{n}, \mathbf{R}^{s}$ и $\mathbf{R}^{q}$ задавалась одна и та же чебышевская норма $l_{\infty}$.

\section{Список литературы}

1. Емеличев В. А., Коротков В. В., О радиусе устойчивости эффективного решения многокритериальной задачи портфельной оптимизации с критериями Сэвиджа. Дискретная математика (2011) 23, №4, 33-38.

2. Emelichev V. A., Korotkov V. V., Post-optimal analysis in a multiple criteria problem of investment risk management based on Markowitz's portfolio theory. In: Abstr. 21st MCDM Intern. Conf. Univ. Jyväskylä, 2011, pp. 50-51.

3. Markowitz H. M., Portfolio selection: Efficient diversification of investments. Wiley, New York, 1959.

4. Царев В. В., Оченка экономической эффективности инвестиций. Питер, Санкт-Петербург, 2004.

5. Виленский П. Л., Лившиц В. Н., Смоляк С. А., Оценка эффективности инвестиционных проектов: теория и практика. Дело, Москва, 2008.

6. Bronshtein E. M., Kachkaeva M. M., Tulupova E. V., Control of investment portfolio based on complex quantile risk measures. J. Computer and Systems Sci. Intern. (2011) 50, 174-180.

7. Wald A., Statistical decision functions. Wiley, New York, 1950.

8. Емеличев В. А., Кричко В. Н., Формула радиуса устойчивости векторной $l_{\infty}$-экстремальной траекторной задачи. Дискретная математика (2004) 16, №1, 14-20. 
9. Гуревский Е. Е., Емеличев В. А., Об устойчивости векторной булевой задачи минимизации абсолютных уклонений от нуля линейных функций. Известия вузов. Математика (2006) №12, 27-32.

10. Емеличев В. А., Кузьмин К. Г., О радиусе устойчивости векторной задачи целочисленного линейного программирования в случае регулярности нормы в критериальном пространстве. Кибернетика и системный анализ (2010) №1, 82-89.

11. Slater M., Lagrange multipliers revisited, Cowles Foundation Discussion Paper 80. Cowles Foundation for Research in Economics at Yale University, 1959.

12. Emelichev V., Podkopaev D., Quantitative stability analysis for vector problems of 0-1 programming. Discrete Optimization (2010) 7, 48-63.

Статья поступила 25.01.2012. 